\title{
GCU
}

Glasgow Caledonian

University

University for the Common Good

\section{Optimal planning of EV charging network based on fuzzy multi-objective optimisation} Qian, Kejun; Gu, Juping; Zhou, Chengke; Yuan, Yue; Zhang, Xinsong; Zhou, Hui

\section{Published in:}

CIRED - Open Access Proceedings Journal

DOI:

10.1049/oap-cired.2017.0551

Publication date:

2017

Document Version

Publisher's PDF, also known as Version of record

Link to publication in ResearchOnline

Citation for published version (Harvard):

Qian, K, Gu, J, Zhou, C, Yuan, Y, Zhang, X \& Zhou, H 2017, 'Optimal planning of EV charging network based on fuzzy multi-objective optimisation', CIRED - Open Access Proceedings Journal, pp. 1-5.

https://doi.org/10.1049/oap-cired.2017.0551

\section{General rights}

Copyright and moral rights for the publications made accessible in the public portal are retained by the authors and/or other copyright owners and it is a condition of accessing publications that users recognise and abide by the legal requirements associated with these rights.

Take down policy

If you believe that this document breaches copyright please view our takedown policy at https://edshare.gcu.ac.uk/id/eprint/5179 for details of how to contact us. 


\title{
Optimal planning of EV charging network based on fuzzy multi-objective optimisation
}

\author{
Kejun Qian ${ }^{1}$, Juping $\mathrm{Gu}^{2}$, Chengke Zhou ${ }^{3}$, Yue Yuan ${ }^{4}$, \\ Xinsong Zhang ${ }^{2}$, Hui Zhou ${ }^{2}$ \\ ${ }^{1}$ Suzhou Power Supply Company, State Grid Corporation of China, Suzhou, People's Republic of \\ China \\ ${ }^{2}$ Nantong University, Nantong, People's Republic of China \\ ${ }^{3}$ Glasgow Caledonian University, Glasgow, UK \\ ${ }^{4}$ Hohai University, Nanjing, People's Republic of China \\ 凶E-mail: kejun.qian@xjtlu.edu.cn
}

\begin{abstract}
Planning for electric vehicle (EV) charging facilities network plays a crucial role in boosting EV numbers, and also has direct impacts on the convenience of EV owners and the operation of distribution systems. Here, mathematical models for EV charging network planning are proposed with objectives to maximise the charging service capacity and to minimise power losses in distribution systems. The model proposed in this paper is a typical multi-objective decision-making model, with two optimisation objectives of different dimensions and also conflict with each other. Membership functions are introduced to transform the original planning model to a single-objective optimisation problem based on the maximum satisfaction degree by fuzzy processing the two optimisation objectives. The proposed model is solved by genetic algorithm. In the end, a 25-node traffic network and an IEEE 33-node distribution system are utilised to verify the models and the solving techniques presented here.
\end{abstract}

\section{Introduction}

With the depletion of fossil fuels and the increasing pollution of the environment in recent years, countries across the world are devoted to deploying electric vehicles (EVs) in the transportation system [1]. Authorities in China introduced a series of preferential policies for EV market. In 2012, the sales of new energy vehicles in China were 12,791, among which fully EVs 11,375 , while in 2013, the sales of EVs reached 17,600, and in 2014, this number exceeded 75,000.

EV charging network plays a crucial role in facilitating charging of EVs, thus will to a large extent affect the convenience of EV owners. From the point of view of power system operator, EV charging is a new load in distribution network, and may affect the temporal and spatial distribution of the distribution system load. An inappropriate planning of EV charging network may have negative effect on the deployment of EVs, worsen the distribution network power quality, and cause significant increase in distribution network losses.

In recent years, an increasing attention has been paid to the optimal planning of EV charging stations, both in industry and academics [2-6]. EV charging network planning consists of determining optimal siting and capacity for charging facilities. In [2], the influence of road network structure, traffic information, and users' travelling habits on the location and capacity of the charging station were considered; the service range of each charging station was divided by the Voronoi diagram. In [3], the subproblem of location and capacity of charging facilities were dealt with separately and the candidate location of charging station was determined by a two-step screening method. In [4], the cost of developing charging stations and the charging services were taken into account simultaneously, and a charging network planning model based on the minimum cost was developed. In [5], a minimised cost model for determining the locations and capacities of charging for EVs is developed considering constraints such as the distances between the substation and candidate locations of EV charging substations, the number of EVs, and the cost of installing charging stations.

The existing work has addressed the basic theory and methods of EV charging network planning. However, a planning model which can not only consider the traffic network, but also the power network and the users' satisfaction is still required. On the basis of the existing research work, this paper considers the EV charging station as a public service facility, and proposes a multi-objective optimisation model, in which, the objective functions are defined to, respectively, maximise the captured traffic network flow, and to minimise the network losses. A genetic algorithm (GA) based on real number coding is used to solve the planning model. Finally, the proposed model and algorithm are validated by a 25 -node traffic network and an IEEE 33-node power distribution system.

\section{Network service capacity model}

The objective of public service facilities such as gas stations, petrol stations, charging stations, and other network services is to provide as many services as possible to all users. At present, the service capacity of the facilities mentioned above is usually described based on the point-based demand model or the path-based demand model.

\subsection{Point-based demand model}

The point demand model is developed on the basis of the P-Median model proposed by Hakimi in 1964 [7]. By optimising the $p$ service facilities, the weighted distance between each demand point and the 
service facility in the network is minimised, with a model shown below [8]

$$
\begin{gathered}
\min \sum_{i} \sum_{j} h_{i} d_{i j} Y_{i j} \\
\text { s.t. } \sum_{j} Y_{i j}=1 \quad \forall i \\
\sum_{j} X_{j}=p \\
Y_{i j}-X_{j} \leq 0 \quad \forall i, j \\
X_{j}=0,1 \quad \forall j \\
Y_{i j}=0,1 \quad \forall i, j
\end{gathered}
$$

where $i$ is the demand point, $j$ the candidate location of the service facility, $h_{i}$ the demand in at $i$, and $d_{i j}$ the distance from $i$ to $j$. If the candidate location $j$ has a service facility, then $X_{j}=1$, otherwise $X_{j}=0$. If the service facility $j$ can meet the demand in $i$, then $Y_{i j}=1$, otherwise $Y_{i j}=0, p$ is the number of service facilities.

The point demand model uses network nodes to represent the demand for charging services in the planning of EV charging network. The largest limitation of this model is that it assumes that the consumer's charging demand is limited to the nodes, while ignoring the demand in the traffic path. In addition, the point demand model hands over all the demand in a certain service area to the charging station at this point, irrespective of its service capability, which is unreasonable.

\subsection{Path-based demand model}

The path-based demand model uses the traffic flow on the path to represent the demand for charging services, and is not limited to the nodes in the network. The objective is to maximise the traffic flow captured by the service facilities. Hodgson [9] first proposed the flow capturing location model (FCLM) in 1990 shown below

$$
\begin{gathered}
\max \sum_{q \in Q} f_{q} y_{q} \\
\text { s.t. } \sum_{i}^{n} x_{i}=p \\
\sum_{i \in N r} x_{i}=y_{q} \quad q \in Q \\
y_{q}, x_{i} \in\{0,1\} \quad q \in Q, i \in N
\end{gathered}
$$

where $y_{q}$ is a binary variable that characterises whether or not the traffic on line $q$ can be captured by the charging network, ' 1 ' means that it can be captured, ' 0 ' means it is not captured, $f_{q}$ is the traffic flow on line $q, x_{i}$ the binary variable representing whether to develop service facilities at node $i$, if yes, $x_{i}=1$, otherwise $x_{i}=0$, and $Q$ the set of all lines in the network.

Compared with the point-based demand model, FCLM can better respond to EVs' charging demand on the path. Therefore, FCLM model is employed in this paper to represent the EV charging demand by captured traffic flow. The more traffic flow captured by the charging network, the larger service capability of the charging network is.

For any traffic network, it can be assumed that it is formed by cross-distribution of a plurality of origin-destination (O-D) lines. The nodes and distances through which the O-D lines pass can be calculated by the Floyd algorithm. The traffic flow on line $q$ can be calculated by the gravity space interaction model [9] shown as below

$$
f_{q}=\frac{W_{\mathrm{O}} W_{\mathrm{D}}}{1.5 d_{q}} \quad q \in Q
$$

where $W_{\mathrm{O}}$ is the weight of the origin node, $W_{\mathrm{D}}$ the weight of the destination node, and $d_{q}$ the length of line $q$.

\section{Mathematical modelling}

\subsection{Objective functions}

3.1.1 Charging service capacity maximisation: Maximisation of the charging service capacity is the primary goal of the EV charging network planning. In this paper, all the traffic flow on $\mathrm{O}-\mathrm{D}$ lines captured by the charging network is used to measure the charging service capacity, i.e. the model has the following objective

$$
\max F_{c}=\sum_{q \in Q} f_{q} y_{q}
$$

It is noted that for the optimisation of EV charging network, the determination of the binary variable $y_{q}$ is related to the maximum travel distance $L$ in the EV full-power state, the distance between the nodes on the line $q$, and the location of the charging station.

3.1.2 Distribution network losses minimisation: From the point of view of distribution network, EV charging demand is a high-power load, whose access to the power grid will result in temporal and spatial changes in the load, thus may cause changes in power flow, which may in turn lead to an increase in network losses. Therefore, to minimise the additional network losses caused by the charging network is also one of the optimisation objectives to be considered in the charging network planning

$$
\begin{gathered}
\min F_{\text {loss }}=\sum_{i=1}^{n} P_{\text {loss }, i} \\
P_{\text {loss }, i}=\frac{P_{i}^{2}+Q_{i}^{2}}{U_{i}^{2}} R_{i}
\end{gathered}
$$

where $P_{\text {loss }, i}$ is the power losses in branch $i, n$ the number of branches, $P_{i}$ the real power at the end of branch $i, Q_{i}$ the reactive power, $U_{i}$ the voltage at the end of branch $i$, and $R_{i}$ the resistance of branch $i$.

\subsection{Constraint conditions}

\subsubsection{Number of charging stations:}

$$
\sum_{i=1}^{n} X_{i}=N
$$

where $X_{i}$ is a binary variable that characterises whether the charging station is developed at node $i$, if yes, $X_{i}=1$, otherwise $X_{i}=0$, and $N$ the number of charging stations to be developed.

\subsubsection{Capacity of the charging station:}

$$
\sum_{i=1}^{n} X_{i} W_{i} \geq W_{\max }
$$

where $W_{i}$ is the capacity of charging station at node $i$ and $W_{\max }$ the max charging demand in the planned area. 


\subsubsection{Voltage deviation:}

$$
\frac{\left|U_{i}-U_{\mathrm{N}}\right|}{U_{\mathrm{N}}} \leq \alpha \quad \forall i
$$

where $U_{i}$ is the voltage at node $i, U_{\mathrm{N}}$ the nominal voltage of distribution network, and $\alpha$ the allowed maximum voltage deviation.

\subsubsection{Power flow on transmission lines:}

$$
P_{l} \leq P_{l, \max }
$$

where $P_{l}$ is the active power of line $l$ and $P_{l, \max }$ is the maximum power that the line $l$ allows to flow.

\subsection{Multi-objective fuzzy processing}

The EV charging network planning model proposed in this paper is a typical multi-objective optimisation problem, which has two optimisation objectives, maximisation of charging service capacity and minimisation of distribution network losses. The two objectives may conflict with each other; therefore, it is difficult to achieve a mutual coordination. In this paper, membership functions are used to fuzzify the two suboptimisation objectives in the model.

The selection of subordinate function is a prerequisite for suboptimisation objective fuzzification; however, it is empirical and so far, there is no unified method to refer. In this paper, the shape function shown in Fig. 1 is selected as the membership function of the suboptimisation objective. As can be seen from the figure, the larger the membership, the more satisfied with the optimisation results by the stakeholders

$$
\begin{gathered}
\mu\left(F_{\mathrm{c}}\right)= \begin{cases}0, & F_{\mathrm{c}} \leq F_{1}-\delta_{1} \\
\frac{F_{\mathrm{c}}-F_{1}+\delta_{1}}{\delta_{1}}, & F_{1}-\delta_{1}<F_{\mathrm{c}} \leq F_{1} \\
1, & F_{\mathrm{c}}>F_{1}\end{cases} \\
\mu\left(F_{\text {loss }}\right)= \begin{cases}\frac{1,}{F_{2}+\delta_{2}-F_{\text {loss }}} \delta_{2}, & F_{2}<F_{\text {loss }} \leq F_{2}+\delta_{2} \\
0, & F_{\text {loss }}>F_{2}+\delta_{2}\end{cases}
\end{gathered}
$$

where $F_{1}$ represents the maximum service capacity of the charging network, i.e. the theoretical maximum traffic flow that can be captured by the charging network, $\delta_{1}$ is the allowed reduction in the captured traffic flow, $F_{2}$ the minimum theoretical value of the distribution network losses, and $\delta_{2}$ the allowed increase in the network losses. The method for determining the above parameters will be explained in detail in Section 3.4.

Once the membership function is obtained, the satisfaction index $\mu$ is defined to represent the satisfaction degree of the stakeholders with the whole planning scheme

$$
\mu=\min \left\{\mu\left(F_{\mathrm{c}}\right), \mu\left(F_{\text {loss }}\right)\right\}
$$
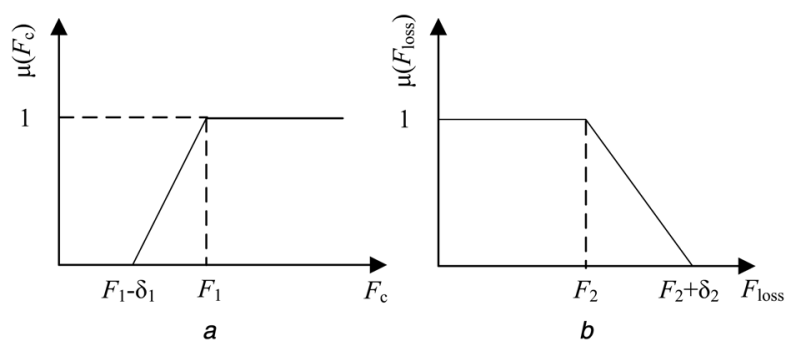

Fig. 1 Membership function

(a) X axis: Membership function of charging service capacity, $\mathrm{Y}$ axis: Satisfaction index, (b) $\mathrm{X}$ axis: Membership function of distribution network losses, $\mathrm{Y}$ axis: Satisfaction index
As a result, the original multi-objective optimisation problem is transformed into a single optimisation problem based on the maximum satisfaction index $\mu$, modelled as follows

$$
\begin{gathered}
\max \mu \\
\text { s.t. }-F_{\mathrm{c}}+\delta_{1} \mu \leq-F_{1}+\delta_{1} \\
F_{\text {loss }}+\delta_{2} \mu \leq F_{2}+\delta_{2} \\
0 \leq \mu \leq 1
\end{gathered}
$$

The above model takes into account the characteristics of the charging station as a public service facility as well as a power facility. The model aims to improve the network service capacity while minimising its impact on the distribution network.

\subsection{GA-based methodology to solve the model}

GA starts from the initial population, through multi-path search optimisation, with an ability to optimise non-linear, discrete, multi-constrained, multi-variable problems. In this paper, GA is employed to solve the proposed EV charging network planning model. First, taking the maximum traffic flow captured by the charging network as the optimisation objective, calculate the maximum traffic flow $F_{\mathrm{c}, 1}$, and the corresponding network losses $F_{\text {loss,1 }}$. Then, taking the minimum network losses as the objective, calculate the network losses $F_{\text {loss,2 }}$, and the traffic flow $F_{\mathrm{c}, 2}$ captured by the charging network. Finally, let $F_{1}=F_{\mathrm{c}, 1}$, $F_{2}=F_{\text {loss }, 2}, \delta_{1}=F_{\mathrm{c}, 1}-F_{\mathrm{c}, 2}, \delta_{2}=F_{\text {loss }, 1}-F_{\text {loss }, 2}$. On the basis of the above work, the EV charging network planning model, in which the satisfaction degree $\mu$ is the optimisation objective, can be solved by GA.

\section{Simulation and results}

\subsection{Test system}

In this paper, a 25-node traffic network and an IEEE 33-node power distribution system as shown in Figs. 2 and 3, respectively, are used to verify the effectiveness of the proposed model. In general, important nodes (such as bus stations, railway stations etc.) in the traffic network are also important load nodes in the distribution network. Therefore, it is assumed that the nodes in the traffic network coincide with the nodes in the distribution network, and for nodes in the distribution system do not coincide with the traffic network, they are ignored when coding the chromosome. The number on the line between two nodes indicates the distance in kilometre.

Set the EV maximum travel distance $L=120 \mathrm{~km}$, the number of charging station to be built $N=4$. There are four kinds of charging station with the capacity of $0.1,0.2,0.3$, and $0.4 \mathrm{MW}$, which are represented by $1,2,3$, and 4 , respectively. The maximum charging service demand $W_{\max }$ is set to be $0.8 \mathrm{MW}$. The maximum allowable voltage deviation $\alpha$ is set to $10 \%$. The weight coefficients of traffic nodes are shown in Table 1 [9].

According to the coding scheme described above, the length of GA chromosome $n=25$, the size of the initial population size is 50 , the maximum evolutionary algebra $G_{\max }=120$, the crossover probability $p_{\mathrm{c}}=0.5$, and the mutation probability $p_{\mathrm{m}}=0.2$.

\subsection{Simulation results analysis}

Table 2 shows the optimisation results when only subobjectives $F_{1}$ and $F_{2}$ are considered.

It can be seen from Table 2 that if only the maximum service capacity of the charging network is considered in the planning, the captured traffic flow reaches the theoretical maximum value $F_{\mathrm{c}, 1}$, which is $0.1148(\sim 37.7 \%$ of the total traffic flow), but at this time the network loss $F_{\text {loss, } 1}$ is very large, which is $243.298 \mathrm{~kW}$. Conversely, if only consider the minimum network losses in the 


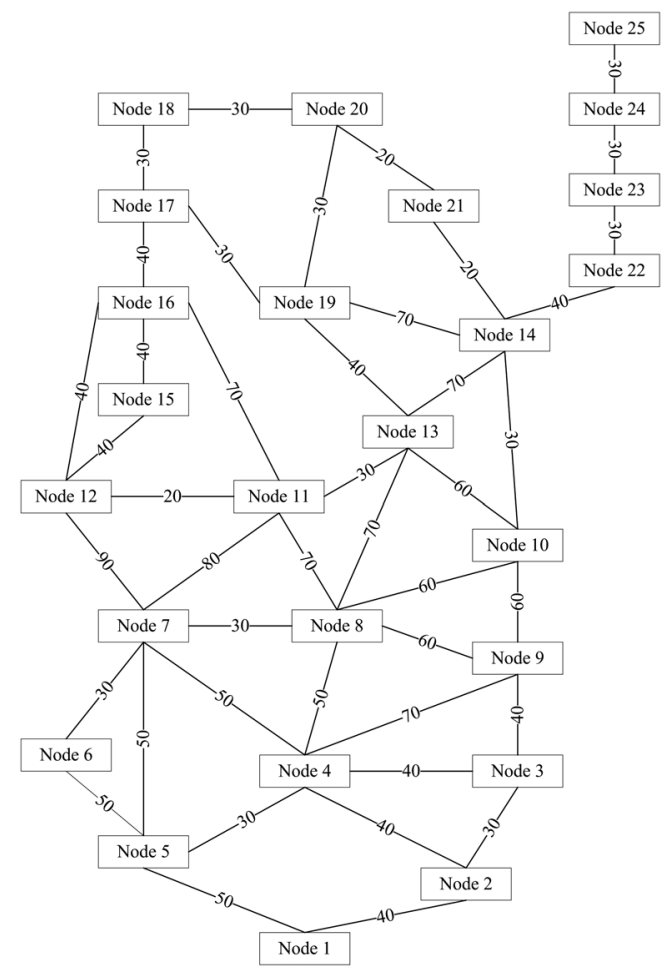

Fig. 2 Twenty-five-node traffic network

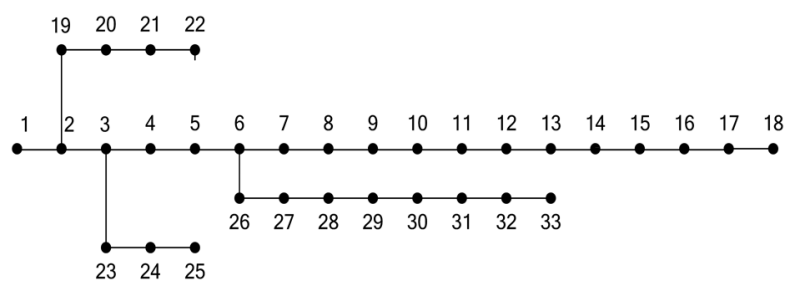

Fig. 3 IEEE 33-node distribution network

Table 1 Weights of various traffic nodes

\begin{tabular}{lccccc}
\hline Node & $\begin{array}{c}\text { Weight } \\
\text { coefficient }\end{array}$ & Node & $\begin{array}{c}\text { Weight } \\
\text { coefficient }\end{array}$ & Node & $\begin{array}{c}\text { Weight } \\
\text { coefficient }\end{array}$ \\
\hline 1 & 0.54 & 10 & 0.54 & 19 & 0.80 \\
2 & 0.80 & 11 & 0.05 & 20 & 0.27 \\
3 & 0.27 & 12 & 0.54 & 21 & 0.27 \\
4 & 0.27 & 13 & 0.05 & 22 & 0.54 \\
5 & 0.27 & 14 & 0.54 & 23 & 0.05 \\
6 & 0.07 & 15 & 0.27 & 24 & 1.34 \\
7 & 0.05 & 16 & 0.27 & 25 & 0.05 \\
8 & 0.54 & 17 & 0.27 & & \\
9 & 0.27 & 18 & 1.07 & & \\
\hline
\end{tabular}

Table 2 Optimisation results when only considering subobjectives

\begin{tabular}{lcccc}
\hline $\begin{array}{l}\text { Optimisation } \\
\text { subobjective }\end{array}$ & $F_{\mathrm{c}}$ & $F_{\text {loss }}, \mathrm{kW}$ & $\begin{array}{c}\text { Charging } \\
\text { facility nodes }\end{array}$ & $\begin{array}{c}\text { Charging } \\
\text { facility type }\end{array}$ \\
\hline $\max F_{\mathrm{c}}$ & 0.1148 & 243.2980 & {$\left[\begin{array}{llll}8 & 14 & 20 & 22\end{array}\right]$} & {$\left[\begin{array}{llll}1 & 1 & 4\end{array}\right]$} \\
$\min F_{\text {loss }}$ & 0.0747 & 207.6273 & {$\left[\begin{array}{llll}1 & 2 & 19 & 22\end{array}\right]$} & {$\left[\begin{array}{llll}4 & 2 & 2 & 1\end{array}\right]$} \\
\hline
\end{tabular}

distribution network, the network losses $F_{\text {loss,2 }}$ can reach the minimum value, also the captured traffic flow $F_{\mathrm{c}, 2}$ will relatively reduce, to only 0.0747 ( $\sim 25.53 \%$ of the total traffic flow). Obviously, the suboptimisation objectives (12) and (13) in the planning model not only have different dimensions, but also

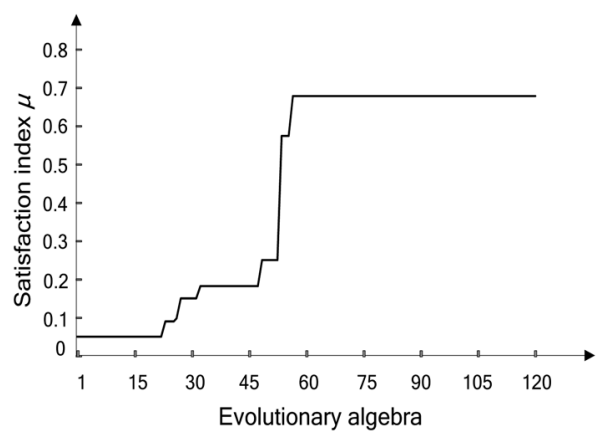

Fig. 4 Algorithm convergence

conflict with each other, making it difficult to achieve optimisation at the same time.

In order to fuzzify the suboptimisation, it is assumed that the allowable traffic flow reduction $\delta_{1}$ is $0.0401\left(\delta_{1}=F_{\mathrm{c}, 1}-F_{\mathrm{c}, 2}\right)$, and the allowable network losses increase $\delta_{2}$ is $35.6707 \mathrm{~kW}$ $\left(\delta_{2}=F_{\text {loss, } 1}-F_{\text {loss, } 2}\right)$. On this basis, two suboptimisation objectives can be fuzzified, thus a single-objective optimisation model is built based on the maximum satisfaction. The satisfaction degree of optimal individual for each evolutionary algebra is shown in Fig. 4. It can be seen from Fig. 4 that the satisfaction degree of the optimal individual increases steadily with the increase in evolutionary algebra, and reaches the optimal value of 0.69 after 60 generations.

Table 3 lists the optimisation results corresponding to the maximum satisfaction. The membership functions of the two

Table 3 Optimisation results

\begin{tabular}{|c|c|c|c|c|c|}
\hline $\begin{array}{l}\mu \\
\left(F_{\mathrm{c}}\right)\end{array}$ & $\left(\begin{array}{c}\mu \\
\left(F_{\text {loss }}\right)\end{array}\right.$ & $F_{\mathrm{c}}$ & $\begin{array}{l}F_{\text {loss }} \\
\mathrm{kW}\end{array}$ & $\begin{array}{c}\text { Charging facility } \\
\text { nodes }\end{array}$ & $\begin{array}{c}\text { Charging facility } \\
\text { type }\end{array}$ \\
\hline 6 & 0.78 & 0.1024 & 215.497 & {$\left[\begin{array}{llll}1 & 19 & 21 & 22\end{array}\right]$} & [2 $\left.\begin{array}{llll}2 & 2 & 2 & 3\end{array}\right]$ \\
\hline
\end{tabular}

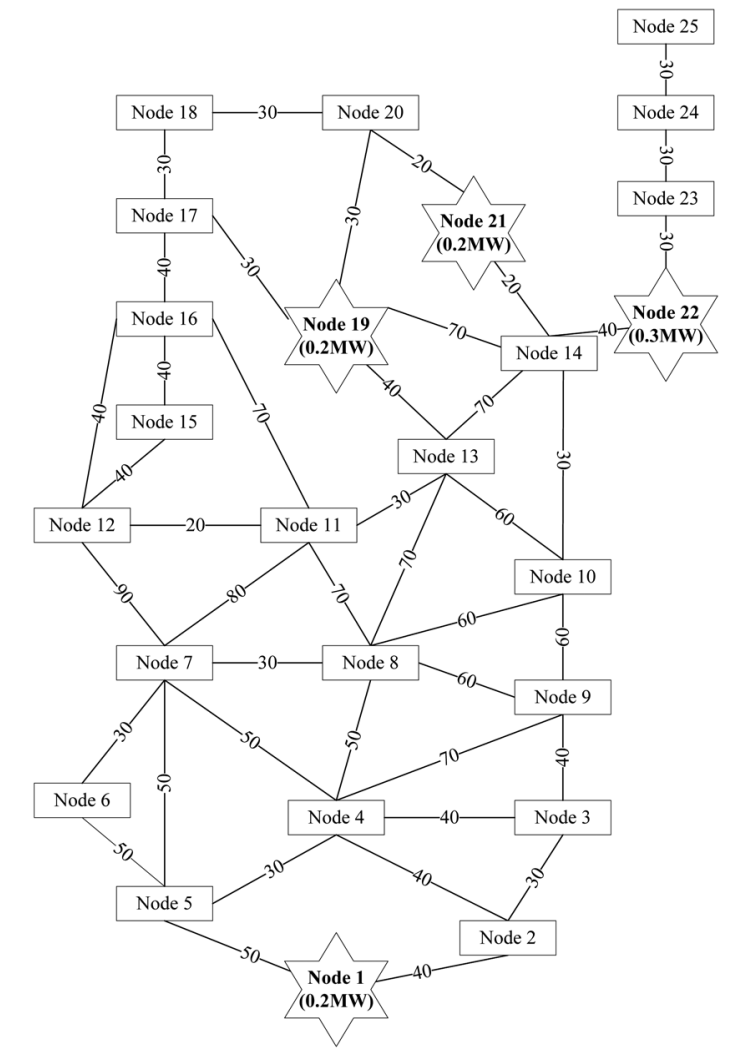

Fig. 5 Fuzzy multi-objective optimisation results 
optimisation objectives are 0.69 and 0.78 , respectively. It can be seen from Table 3 that the optimal results are obtained when both the maximum service capacity of the charging network and the minimum network losses are taken into account. Under this condition, the traffic flow captured by the charging network decreases from the theoretical maximum 0.1148 to 0.1024 , with a percentage of $10.8 \%$, while the network losses decrease from 243.2980 to $215.497 \mathrm{~kW}$, with a percentage of $11.39 \%$.

Fig. 5 shows the EV charging network development scheme considering both the maximum charging service capacity and the minimum network losses. The proposed method considers the dual characteristics of the charging station as a public service facility and a power facility, which gives a reasonable optimisation scheme.

\section{Conclusions and future work}

This paper proposes an EV charging network planning model based on multi-objective optimisation, taking into account both the optimisation of service capacity and the network losses. The paper has the following conclusions:

- The proposed method considers dual characteristics of the charging station as a public service facility and a power facility, which gives a more reasonable optimisation result than the cases only subobjectives are taken into account.

- Test results show that the proposed model can maximise the charging service while minimising the distribution network losses. As a result, stakeholders can make a reasonable decision after being informed the trade-off solution.
- GA has a good convergence when solving the multi-objective optimisation model of EV charging network.

The methodology could be improved in the future work by using more sophisticated models for forecasting EV numbers and determining the EV parking density.

\section{References}

1 Mehar, S., Senouci, S.: 'An optimization location scheme for electric charging stations'. Smart Communications in Network Technologies Conf., Paris, France, 17-19 June 2013, pp. 1-5

2 Ge, S., Feng, L., Liu, H., et al.: 'An optimization approach for the layout and location of electric vehicle charging stations', Elect. Power, 2012, 45, (1), pp. $96-101$

3 Liu, Z., Wen, F., Ledwich, G.: 'Optimal planning of electric-vehicle charging stations in distribution systems', IEEE Trans. Power Deliv., 2013, 28, (1), pp. $102-110$

4 Feng, C., Zhou, B., Lin, N., et al.: 'Electric vehicle charging station planning based on multiple-population hybrid genetic algorithm', Proc. CSU-EPSA, 2013, 25, (6), pp. $123-129$

5 Kou, L.F., Liu, Z.F., Zhou, H.: 'Modeling algorithm of charging station planning for regional electric vehicle', Mod. Elect. Power, 2010, 27, (4), pp. 44-48

6 Trivedi, A., Menon, B., Srinivasan, D., et al.: 'Multi-objective siting and sizing of E charging stations in the distribution system'. 2015ISGT ASIA, Bangkok, 2015, pp. 1-6

7 Upchurch, C., Kuby, M.: 'Comparing the P-median and flow-refueling models fo locating alternative-fuel stations', J. Transp. Geogr., 2010, 18, (6), pp. 750-758

8 Hakimi, S.L.: 'Optimum locations of switching centers and the absolute centers and medians of a graph', Oper. Res., 1964, 12, pp. 450-459

9 Hodgson, M.J.: 'A flow capturing location-allocation model', Geogr. Anal., 1990, 22, (3), pp. 270-279 